\title{
Anesthetic Management of a Patient with Mitochondrial Encephalopathy, Lactic Acidosis, and Stroke-Like Symptoms (MELAS): A Case Report
}

\section{Refaat Shehab $^{1 *}$ and MO Ababneh ${ }^{2}$}

${ }^{1}$ Senior Consultant, Anaesthesiologist, Abdali Medical Centre, Al-Istethmar

Street - Abdali Boulevard - Amman, Jordan

${ }^{2}$ Consultant, Clinical Director, Anaesthesia Department, Abdali Medical Centre, Al-Istethmar Street - Abdali Boulevard - Amman, Jordan

*Corresponding Author: Refaat Shehab, Senior Consultant, Anaesthesiologist, Abdali Medical Centre, Al-Istethmar Street - Abdali Boulevard - Amman, Jordan.
Received: August 27, 2021

Published: September 14, 2021

(C) All rights are reserved by Refaat Shehab and MO Ababneh.

\section{Abstract}

Mitochondrial encephalopathy, lactic acidosis, stroke-like symptoms (MELAS) syndrome is a specific mitochondrial myopathy that results in defects in respiratory enzyme complexes I and IV.

This ultimately causes defects in aerobic metabolism, especially in high-energy-requiring organs, leading to an obvious concern with anesthetic management.

Mitochondrial disease (MD) is recognized as an important cause of a wide range of physiologic changes that affect the perioperative period [1], strongly affected by anesthetics.

We managed a 36-year male patient with MELAS, underwent a ureteroscopy, lithotripsy and indwelling double J stent exchange. The patient was successfully managed with a spinal/epidural anaesthesia.

Keywords: MELAS; Anesthetic Management; Myopathy; Genetics

\section{Introduction}

Mitochondrial myopathies (MMs) represent a wide range of defects in the mitochondria. MELAS syndrome is a type of mitochondrial disease that is systemic in nature. This is because of the multiple function's mitochondria serve in oxidative phosphorylation. MELAS has been speculated to affect respiratory enzyme complexes I and IV, which are essential in converting substrates from glycolysis, fatty acid oxidation, and the tricarboxylic acid cycle to ATP [2].
MELAS involves a mitochondrial defect in aerobic metabolism, the disease has implications for anesthesia management. Anesthetic medications, surgery itself, and fasting in preparation for surgery can all increase the metabolic burden, leading to possible exacerbations in tissues dependent on large amounts of oxygen, such as the heart, muscle, and central nervous system [3]. MMs have also been speculatively linked to malignant hyperthermia.

Given the relatively small published experience of this vulnerable population, both general and regional anaesthesia has been

Citation: Refaat Shehab and MO Ababneh. "Anesthetic Management of a Patient with Mitochondrial Encephalopathy, Lactic Acidosis, and Stroke-Like Symptoms (MELAS): A Case Report". Acta Scientific Cancer Biology 5.10 (2021): 04-07. 
reported in this group of patients successfully, we chose regional anaesthesia for our case, as to minimize untoward responses to many medications used in general anaesthesia.

\section{Case Summary}

A 36-year-old male, presented with a left ureter indwelling double J stent (4 months), post extracorporeal shock wave lithotripsy (ESWL), and several left renal stones. The patient's other medical conditions included hearing loss, visual impairment and sever muscle wasting, wheelchair and continuous positive airway pressure CPAP dependent for the last 5 years.

His surgical history includes; appendicectomy at age of 15 years under general anaesthesia, stem cells implant, one year ago and double J stent insertion 6 months back under spinal anaesthesia.

The patient's medications included, Co-enzyme Q10 $100 \mathrm{mg}$, vitamin B3, Candesartan $4 \mathrm{mg}$ daily and Bisoprolol 1.5mg twice daily. He was assessed 5 days prior surgery, in the perioperative medicine clinic, anesthesia options, risks and postoperative care, all has been discussed at length.

On examination the patient was in a wheelchair, looks thin, unable to shake hands, $45 \mathrm{~kg}$ in weight, $152 \mathrm{~cm}$ in height, using all his accessory muscles for breathing, couldn't finish a sentence, had a blood pressure of 103/57, a pulse rate of $90 \mathrm{bpm}$ and a respiratory rate of $24 \mathrm{bpm}$. His American Society of Anesthesiologists Classification was designated as class III.

His investigations include, serum complete blood count, kidney function, blood glucose level, ammonia level, serum electrolytes and coagulation studies were all within normal values. Left ventricular ejection fraction estimated at $50 \%$ as he was diagnosed earlier by the cardiologist with a cardiomyopathy.

The plan of anaesthesia was discussed at length with the patient and his family, in the preoperative clinic, type of anaesthesia, intraoperative, postoperative course, probable complications and options.

In the view of the case, patient was consented for spinal/epidural anaesthesia with no sedation (although the surgeon expected time was 3 hour), to minimize polypharmacy, possible conversion to general anaesthesia if needs to be, possible intensive care admis- sion and/or ventilation postoperatively, and advised to bring in his own CPAP device with him.

In the operating room, an intravenous access was secured with an 18G cannula, routine monitors were applied plus left radial intraarterial catheter was inserted under local anaesthesia, patient was placed in his left lateral position, under aseptic technique local anaesthesia infiltration at L3/L4, (B Braun) 22G Quincke spinal needle was used, $1.75 \mathrm{ml}$ Bupivacaine (hyperbaric) mixed in 20micg Fentanyl was injected intrathecally, followed by L4/L5 18G Tuohy epidural (Portex)needle, 3ml of a mixture of Bupivacaine $1 \%$, Fentanyl $2 \mathrm{micg} / \mathrm{ml}$ was given, followed by continuous infusion of the same mixture at a rate of $2 \mathrm{ml} /$ hour.

Block was checked to be satisfactory, the operation lasted three and half hours, on high flow nasal cannula, forced air warming blanket (Bear Hugger) was applied, patient was comfortable all through, did not the CPAP intraoperatively, sedation was avoided, and fluid of warmed normal saline was given in total $750 \mathrm{ml}$. patient remained stable all through and normal body temperature.

Post-operatively the patient was transferred to the recovery room, fully monitored for two hours, epidural infusion was continued till next day at the same rate, arterial line was disconnected then transferred to the ward with reasonable muscle power, satisfactory breathing pain free.

\section{Discussion}

Mitochondrial Encephalopathy Lactic Acidosis, and stroke-like episodes (MELAS) is the most common subtype of mitochondrial encephalopathies, the onset of illness is often in early adulthood. Mitochondria are the "energy factory" of our body, they process oxygen and convert substances into energy, producing almost $90 \%$ of the energy needed for the body.

The hallmarks of MELAS are encephalomyopathy with seizures and/or dementia, lactic acidosis, and recurrent stroke-like episodes. These episodes are not typical strokes, which are interruptions in the brain's blood supply that cause sudden neurological symptoms. However, the episodes can produce stroke-like symptoms in the short term (such as temporary vision loss, difficulty speaking, or difficulty understanding speech) and lead to progressive brain injury. The cause of the stroke-like episodes is unclear. 
Patients with mitochondrial cytopathies results in decreased ATP production, insufficient lipid metabolism and formation of damaging free radicals [4], this might cause the anaesthtics to affect patients with mitochondrial disease by causing further decrease in energy supplies [5]. Mitochondrial myopathies have also been speculatively linked to malignant hyperthermia owing to the similar presentation of symptoms. Controversy exists, however, over whether anesthetic management should be based on concerns to avoid malignant hyperthermia triggers.

Our patient had a stroke like activity manifested by seizures, followed by hearing loss, vision loss, CPAP dependent nighttime and cardiomyopathy with an ejection fraction (EF) of 50\%. The diagnosis of MELAS was confirmed at age of 30 years. He underwent stem cells trial 7 months back, with minimal improvement, mainly in supporting his head. He is intellectual and aware of his condition with vast knowledge.

In the pre-assessment clinic a week before surgery, we have discussed at length with his family, type of anaestheia (unfortunately his younger brother has been diagnosed with the same syndrome recently), he was aware of all side effects, complications of each type of anaesthtic, moreover, he went to united states for consultation, and was advised to have spinal anaesthesia. Due to predict length of surgery by our surgeon we thought spinal/epidural will be more suitable for him and suggested to keep the epidural catheter for 24 hours, to minimize systemic analgesia side effects.

In reviewing a limited number of reports available, describing adverse events and outcomes in patients with mitochondrial diseases following surgery and anaesthesia, a few inferences can be made. However, these does not define the limits for the safe use of any anaesthetic in these cases

MELAS patients have increased susceptibility to lactic acidemia under increased metabolic stress prolonged fasting, hypoglycemia, postoperative nausea and vomiting, hypothermia, acidosis, and hypovolemia should be avoided as they might cause a metabolic burden [6].

Choice of fluids in MELAS patients remains controversial because whereas hypoglycemia can cause increased metabolic burden, glucose-containing fluids may not be optimal for avoidance of seizures [7]. recommendations from Malignant Hyperthermia Association of the United States (MHAUS), state that avoidance of volatile anesthetics is not necessary because of no increased susceptibility of patients with MMs to malignant hyperthermia [8]. Avoidance of succinylcholine has been discussed because of one case report in 1985 in which life-threatening hyperkalemia occurred in a patient with mitochondrial dysfunction after its administration. However, just as MHAUS suggests, no definitive genetic link has been shown between malignant hyperthermia and mitochondrial diseases such as MELAS [9].

In a retrospective review of 64 patients with mitochondrial disease ( 6 cases of MELAS), a variety of anesthetic techniques were used with no significant events such as unanticipated hospital admission, cardiac arrest, hypothermia, hyperthermia, prolonged post-anesthesia stay, increased lactic acidosis, or metabolic decompensation after exposure [10].

Multiple case reports have also documented the successful administration of regional anesthetics in MELAS patients without adverse effects, including spinal, combined general endotracheal anesthesia with epidural anesthesia, epidural anesthesia for postoperative pain, and epidural catheter placement for labor analgesia [11].

Postoperative considerations must include possible metabolic disturbances, postoperative respiratory failure, and adequate analgesia to prevent acidosis. Other considerations can include adequate control of nausea and vomiting and postoperative shivering. These patients should be monitored in the intensive care unit because of the potential for metabolic disturbances.

\section{Conclusion}

Taking all modes of anaesthesia into consideration we feel that the optimum precautions to decrease the risk of complications in these cases, by reducing the metabolic burden in MELAS patients by avoidance of: lactate in intravenous fluids, hypothermia, prolonged fasting, and postoperative nausea vomiting, early intervention in case of respiratory deterioration and heightened level of suspicion for pneumonia.

\section{Conflicts of Interest}

None declared. 


\section{Bibliography}

1. Muravchick S and Levy RJ. "Clinical implications of mitochondrial dysfunction”. Anesthesiology 105.4 (2006): 819-837.

2. Falk MJ and Sondheimer N. "Mitochondrial genetic diseases". Current Opinion in Pediatrics 22.6 (2010): 711-716.

3. Finsterer J. "Central nervous system manifestations of mitochondrial disorders". Acta Neurologica Scandinavica 114.4 (2006): 217-238.

4. Codier E and Codier D. "Understanding mitochondrial disease and goals for its treatment". The British Journal of Nursing 23.5 (2014): 254-260.

5. Online Mendelian Inheritance in Man (2015).

6. Petty RKH., et al. "The clinical features of mitochondrial myopathy". Brain 109 (1986): 915-938.

7. Wallace JJ., et al. "Anaesthesia and mitochondrial disease". Paediatric Anesthesia 8 (1998): 249-254.

8. Malignant Hyperthermia Association of the United States. Does mitochondrial myopathy (MM) increase an individual's susceptibility to malignant hyperthermia (MH)? Malignant Hyperthermia Association of the United States website (2015).

9. RJ Dandurand., et al. "Mitochondrial disease. Pulmonary function, exercise performance, and blood lactate levels". Chest 108.1 (1995): 182-189.

10. D’Ambra MN., et al. "Kearns-Sayer syndrome and pancuroniumsuccinylcholine-induced neuromuscular blockade". Anesthesiology 51 (1979): 343-345.

11. Rosaeg OP., et al. "Anaesthesia management of labour and delivery in parturient with mitochondrial myopathy". Canadian Journal of Anesthesia 43 (1996): 403-407.

\section{Volume 5 Issue 10 October 2021}

\section{(C) All rights are reserved by Refaat Shehab} and MO Ababneh. 